\title{
IDEAL THEORY IN TWO-DIMENSIONAL REGULAR LOCAL DOMAINS AND BIRATIONAL EXTENSIONS
}

\author{
William Heinzer and David Lantz
}

Department of Mathematics, Purdue University, West Lafayette, IN 47907. E-mail: heinzer@math.purdue.edu

Department of Mathematics, Colgate University, 13 Oak Drive, Hamilton, NY 13346-1398. E-mail: dlantz@center.colgate.edu

\section{Introduction.}

(0.1) Let $(R, \mathbf{m})$ be a two-dimensional regular local domain with infinite residue field $R / \mathbf{m}$. Associated to an $\mathbf{m}$-primary ideal $I$ in $R$ is its Hilbert polynomial

$$
P_{I}(n)=e_{0}(I)\left(\begin{array}{c}
n+1 \\
2
\end{array}\right)-e_{1}(I) n+e_{2}(I),
$$

the integer-valued polynomial giving the length of the $R$-module $R / I^{n}$ for sufficiently large positive integers $n$. The coefficient $e_{0}$ is well known to be a positive integer, the multiplicity of $I$, and in our context, the coefficients $e_{1}$ and $e_{2}$ are known to be nonnegative integers.

A well-known result of Rees [Re1, Theorem 3.2] implies that for each $\mathbf{m}$ primary ideal $I$ of $R$ the integral closure of $I$ is the unique largest ideal containing $I$ and having the same multiplicity. A result of Shah in [Sh, Theorem 1] implies the existence of a unique largest ideal $I_{\{1\}}$ containing $I$ 
and having the same coefficients $e_{0}$ and $e_{1}$ of its Hilbert polynomial. We call $I_{\{1\}}$ the $e_{1}$-ideal associated with $I$. If $I=I_{\{1\}}$, we call $I$ a first coefficient ideal or an $e_{1}$-ideal.

There is an interplay between the internal structure of the ideals in $R$ and the external structure of certain birational extensions of $R$. In this connection, for an $\mathbf{m}$-primary ideal $I$, the blowup of $I$,

$$
\mathcal{B}(I)=\operatorname{Proj}(R[I t])=\left\{R[I / a]_{P}: a \in I-0, P \in \operatorname{Spec}(R[I / a])\right\},
$$

is the projective model over $R$ (in the sense of [ZS, page 120]) consisting of the local domains containing $R$ that are minimal with respect to domination among all the local domains containing $R$ in which the extension of $I$ is principal. There is a nonempty finite subset of $\mathcal{B}(I)$ consisting of local domains in which $I$ generates an ideal primary for the maximal ideal; each of these local domains is one-dimensional and their intersection $D$ is a one-dimensional semilocal domain called the first coefficient domain of $I$. As noted in [HJL, (1.3) and (3.2)], we have $I D \cap R=I_{\{1\}}$; indeed, since all powers $I^{n}$ of $I$ have the same blowup, we have $I^{n} D \cap R=\left(I^{n}\right)_{\{1\}}$, for each positive integer $n$.

Our goal in this paper is a better understanding of $e_{1}$-ideals and their first coefficient domains over a two-dimensional regular local domain. The situation where the first coefficient domain is a semilocal PID is well understood in view of the Zariski theory concerning complete ideals and prime divisors on $R$ (see, e.g., [Z], [ZS, Appendix 5] or [Hu]). In particular, if $V$ is a DVR birationally dominating $R$ which is a spot over $R$ (i.e., in Zariski's terminology a prime divisor of the second kind on $R$; in [A2] a hidden prime divisor of $R$ ), then the ideals of $R$ contracted from $V$ form a descending chain $\mathbf{m}=\mathbf{a}_{0}>\mathbf{a}_{1}>\mathbf{a}_{2}>\ldots$ of complete $\mathbf{m}$-primary ideals, the valuation ideals of $R$ with respect to $V$. The Zariski theory associates to the prime divisor of the second kind $V$ a unique simple (i.e., not factorable into a product of proper ideals) complete ideal $\mathbf{b}$. One way of characterizing $\mathbf{b}$ is that $\mathbf{b}$ is maximal among $\mathbf{m}$-primary ideals $\mathbf{c}$ of $R$ with the property that all powers of $\mathbf{c}$ are contracted from $V$. We have $\mathbf{b}=\mathbf{a}_{n}$ for some $n$. For example, $\mathbf{b}=\mathbf{m}$ if and only if $V$ is the ord-valuation domain $R[y / x]_{\mathbf{m} R[y / x]}$ where $\mathbf{m}=(x, y) R$. If $n>0$, then certain of the ideals $\mathbf{a}_{0}, \ldots \mathbf{a}_{n-1}$ are also 
simple complete ideals. If we label the simple complete ideals in this chain as $\mathbf{b}_{0}=\mathbf{m}, \mathbf{b}_{1}, \ldots, \mathbf{b}_{s}=\mathbf{b}=\mathbf{a}_{n}$, then Zariski proves that each of the valuation ideals $\mathbf{a}_{i}, i \geq 0$, is a product of powers of $\mathbf{b}_{0}, \ldots, \mathbf{b}_{s}$ [ZS, page 392]. For example, if $\mathbf{m}=(x, y) R$ and $V$ is the integral closure of $R\left[x^{2} / y^{3}\right]_{\mathbf{m} R\left[x^{2} / y^{3}\right]}$, then $\mathbf{b}=\left(x^{2}, x y^{2}, y^{3}\right) R$ is the simple complete ideal associated to $V$, and $\mathbf{m}=\mathbf{a}_{0}>\mathbf{a}_{1}=\mathbf{b}_{1}=\left(x, y^{2}\right) R>\mathbf{a}_{2}=\mathbf{m}^{2}>\mathbf{b}$ is the beginning of the chain of ideals $\left\{\mathbf{a}_{i}\right\}$ of $R$ contracted from $V$. The result of Zariski just mentioned implies that each $\mathbf{a}_{i}$ is a power product of $\mathbf{m}, \mathbf{b}_{1}$ and $\mathbf{b}$. More detailed information as to which products of the $\mathbf{b}_{j}$ are actually contracted from $V$ is given by Noh in [No, Theorem 3.1].

(0.2) To describe the same situation from a different starting point, let $I$ be a complete $\mathbf{m}$-primary ideal of $R$. The first coefficient domain $D$ of $I$ is then a semilocal PID which is the intersection of the Rees valuation domains of $I$, i.e., the DVR's on $\mathcal{B}(I)$ that dominate $R$. In this case, $D$ is uniquely determined as the largest one-dimensional semilocal subdomain $E$ of the fraction field of $R$ having the property that all the powers of $I$ are contracted from $E$ (see (3.4) below). If $V_{1}, \ldots, V_{n}$ are the Rees valuation domains of $I$, then the Zariski theory implies that $I$ has the form

$$
\mathbf{b}_{1}^{r_{1}} \ldots \mathbf{b}_{n}^{r_{n}}
$$

where the $r_{j}$ are positive integers and $\mathbf{b}_{j}$ is the simple complete ideal of $R$ associated to $V_{j}, j=1, \ldots, n$.

In the present paper we pursue the study of $e_{1}$-ideals and first coefficient domains begun in [HJL]. In particular, we consider implications of the Zariski theory for these broader classes of ideals and integral domains. Our objective, only partially realized, is to identify the first coefficient domains over a two-dimensional regular local domain and the ideals of which they are first coefficient domains.

In Section 1 we illustrate with several examples properties that onedimensional spots birationally dominating a two-dimensional regular local domain may have or fail to have. We also observe in Proposition 1.1 that the condition of being a spot descends from an integral extension. 
In Section 2 we consider implications of residual transcendence. As part of Theorem 2.2, we prove that if $R$ is a two-dimensional RLR of characteristic $p>0$ with algebraically closed residue field and $D$ is a one-dimensional local domain birationally dominating $R$ such that the integral closure of $D$ is a prime divisor on $R$, then $D$ is the first coefficient domain of an ideal of $R$.

In Section 3 we examine asymptotic behavior of ideals and implications for first coefficient domains. Suppose $(R, \mathbf{m})$ is a local domain that is the intersection of its localizations at height-one primes and $D$ is a one-dimensional semilocal domain birationally dominating $R$. In Theorem 3.3 we prove that if $J$ is an $\mathbf{m}$-primary ideal of $R$ such that $J D$ is principal and $J^{n} D \cap R=J^{n}$ for each positive integer $n$, then the first coefficient domain of $J$ is a localization of $D$. In particular, if $D$ is local, then $D$ is the first coefficient domain of $J$.

As usual, we abbreviate "regular local domain" by RLR and "rank-one discrete valuation domain" by DVR. The words "local" and "semilocal" include the hypothesis of Noetherian. The symbol $<$ between sets denotes proper inclusion. For an ideal $I$ in a Noetherian domain $R$ the blowup of $I$ and the first coefficient domain of $I$ are defined as in (0.1) above. The Rees valuation domains of $I$ are the localizations of the integral closure of the first coefficient domain of $I$ at its maximal ideals. It is convenient to extend some familiar terminology to the case of rings that are not necessarily Noetherian or that have more than one maximal ideal: A ring $D$ containing a domain $R$ having a unique maximal ideal $\mathbf{m}$ is said to birationally dominate $R$ if $D$ is contained in the fraction field of $R$ and for each maximal ideal $N$ of $D$, $N \cap R=\mathbf{m}$. An extension ring $D$ of a ring $R$ is said to be affine over $R$ if $D$ is finitely generated as an algebra over $R$. We say that a ring $D$ with finitely many maximal ideals is a semispot over a subring $R$ if $D$ is a ring of fractions of a ring containing and affine over $R$. If such a $D$ has only one maximal ideal, then we call it a spot over $R$.

\section{One-dimensional birational spots.}

We are interested in considering one-dimensional semilocal domains $D$ that birationally dominate a two-dimensional RLR $R$. A DVR $V$ birationally 
dominating $R$ is a spot over $R$ if and only if the the residue field of $V$ is not algebraic as an extension of $R / \mathbf{m}$ [A1, Proposition 3, page 336]. An interesting property of such a DVR $V$ (also proved in [A1]) is that the residue field $F$ of $V$ is ruled as an extension field of $R / \mathbf{m}$, i.e., $F$ is obtained as a simple transcendental extension of a field intermediate between $R / \mathbf{m}$ and $F$. In view of the fact that $R$ is a two-dimensional RLR, it follows that $F$ is a simple transcendental extension of a finite algebraic extension of $R / \mathbf{m}$.

In general, if $D$ is a one-dimensional semilocal domain birationally dominating $R$, then the integral closure $D^{\prime}$ of $D$ is a semilocal PID birationally dominating $R$. If $R$ is complete, then $D^{\prime}$ is necessarily a semispot over $R$; but for certain $R$ (such as $R=k[x, y]_{(x, y) k[x, y]}$ where $x, y$ are indeterminates over the field $k$ ) there exist DVR's birationally dominating $R$ that are not spots over $R$ (cf., e.g., [HRS]).

We begin by proving a result (Corollary 1.3) that implies that if $D$ is a one-dimensional semilocal domain birationally dominating a two-dimensional RLR $R$ and if the integral closure $D^{\prime}$ of $D$ is a semispot over $R$, then $D$ is a semispot over $R$ and $D^{\prime}$ is a finitely generated $D$-module.

Proposition 1.1. Let $R$ be a Noetherian ring, and let $V$ be a semispot over $R$. Suppose $R \subseteq D \subseteq V$ with $D$ quasilocal and $V$ integral over $D$. Then $D$ is a spot over $R$ and $V$ is a finitely generated $D$-module.

Proof. Since $V$ is a semispot over $R$, there exist elements $a_{1}, \ldots, a_{n} \in V$ such that $V$ is a ring of fractions of $R\left[a_{1}, \ldots, a_{n}\right]$. Let $b_{1}, \ldots, b_{m}$ be the coefficients of monic polynomials over $D$ satisfied by $a_{1}, \ldots, a_{n}$; set $B=R\left[b_{1}, \ldots, b_{m}\right]$ and $A=B\left[a_{1}, \ldots, a_{n}\right]$. Let $Q$ be the center of $D$ on $B$, and let $A_{1}$ and $B_{1}$ be the rings of fractions of $A$ and $B$ at the multiplicative set $B-Q$. Then $B_{1}$ is local, with maximal ideal $Q_{1}=Q B_{1}$, and $A_{1}$ is a finite integral extension of $B_{1}$. Hence $A_{1}$ has only finitely many maximal ideals. Let $P_{1}, \ldots, P_{r}$ denote the centers on $A_{1}$ of the maximal ideals of $V$, and let $S=A_{1}-\left(\bigcup_{i=1}^{r} P_{i}\right)$. Since $V$ is a ring of fractions of $R\left[a_{1}, \ldots, a_{n}\right]$, we have $S^{-1} A_{1}=V$. Choose $a \in S$ such that $a$ is in each maximal ideal of $A_{1}$ distinct from $P_{1}, \ldots, P_{r}$ (if any - otherwise let $a=1$ ). Then $1 / a$ is in $V$ and hence is integral over $D$. Let $c_{1}, \ldots, c_{p}$ be the coefficients of a monic polynomial over $D$ satisfied by 
$1 / a$; let $\left(B_{2}, Q_{2}\right)$ be the localization of $B_{1}\left[c_{1}, \ldots, c_{p}\right]$ at the center of $D$ on this ring, and set $A_{2}=B_{2}\left[1 / a, A_{1}\right]$.

We claim that $A_{2}=V$. To see this, it suffices to show each $s$ in $S$ is a unit in $A_{2}$ : Assume by way of contradiction that $s$ in $S$ is in a maximal ideal $M$ of $A_{2}$. Since $A_{2}$ is integral over $B_{2}$, we have $M \cap B_{2}=Q_{2}$, and so $Q_{1}=M \cap B_{1}=\left(M \cap A_{1}\right) \cap B_{1}$. Since $A_{1}$ is integral over $B_{1}, M \cap A_{1}$ is maximal in $A_{1}$. Moreover, $M \cap A_{1}$ survives in $A_{2}$, so our choice of $a$ assures that $M \cap A_{1}$ is the center on $A_{1}$ of one of the maximal ideals of $V$. But this yields $s \in S \subseteq A_{1}-\left(M \cap A_{1}\right)$, a contradiction.

Therefore, $V$ is an affine extension of $B_{2}$ and hence a finitely generated $D$-module. Thus, by Artin-Tate [Ku, Lemma 3.3, page 16], $D$ is an affine extension of $B_{2}$ and hence a spot over $R$.

To extend this result to the case where $D$ has finitely many maximal ideals, we use:

Proposition 1.2. Let $R$ be an integral domain. Suppose $D$ is an extension domain of $R$ having only finitely many maximal ideals $N_{1}, \ldots, N_{r}$ and having the property that $D_{N_{i}}$ is a spot over $R$ for each $i=1, \ldots, r$. Then $D$ is a semispot over $R$.

Proof. For each maximal ideal $N_{i}$ of $D$ there is a finite subset $T_{i}$ of $D_{N_{i}}$ such that $D_{N_{i}}$ is a localization of $R\left[T_{i}\right]$. And there is an element $s_{i}$ of $D-N_{i}$ for which $s_{i} T_{i} \subseteq D$. Let $A=R\left[\left(\bigcup_{i=1}^{r} s_{i} T_{i}\right) \cup\left\{s_{1}, \ldots, s_{r}\right\}\right]$. If $P_{i}$ denotes the center of $D_{N_{i}}$ on $A$, then $A \subseteq D \subseteq D_{N_{i}}=A_{P_{i}}$; so $D$ is the ring of fractions of $A$ at the complement of the union of the $P_{i}$ 's.

As an immediate corollary of Propositions 1.1 and 1.2, we have:

Corollary 1.3. Let $D$ be a semilocal extension domain of a Noetherian domain $R$, and let $V$ be a domain integral over $D$. If $V$ is a semispot over $R$, then $D$ is also a semispot over $R$.

(1.4) It follows from Corollary 1.3 that a one-dimensional semilocal domain $D$ that birationally dominates a two-dimensional RLR $R$ is a semispot over $R$ if and only the integral closure of $D$ is an intersection of prime divisors of 
the second kind on $R$, or equivalently, if and only if each DVR birationally containing $D$ is a prime divisor of the second kind on $R$.

We are interested in the question of which one-dimensional semilocal domains birationally dominating $R$ are first coefficient domains of ideals of $R$. The first coefficient domains of complete ideals of $R$ are well understood. They are precisely the one-dimensional semilocal PID's birationally dominating $R$ that are semispots over $R$. Moreover, if $I$ and $J$ are complete m-primary ideals of $R$ with first coefficient domains $D_{I}$ and $D_{J}$, respectively, then $D_{I} \cap D_{J}$ is a PID semispot over $R$ and is the first coefficient domain of $I J$. More generally, by the Theorem on Independence of Valuations (e.g., [N, (11.11)] or [ZS, Theorem 18, p. 45]) the intersection of two semilocal PID's birationally dominating a local domain is again a semilocal PID birationally dominating the local domain. But for arbitrary $\mathbf{m}$-primary ideals $I$ and $J$ of $R$, the relation of $D_{I}$ and $D_{J}$ with the first coefficient domain of $I J$ is more delicate. It is not necessarily $D_{I} \cap D_{J}$; indeed, in Example 1.5 we show that $D_{I} \cap D_{J}$ need not be a first coefficient domain of $R$. In this example we make use of the description of the first coefficient domain of an ideal generated by a regular sequence given in [HJL, (3.8)].

Example 1.5. Let $k$ be a field of characteristic 0 and $x, y$ be indeterminates over $k$; set $R=k[x, y]_{(x, y)}$. Then the first coefficient domains of the ideals $\left(x^{2}, y^{2}\right) R$ and $\left(x^{2}, x y+y^{2}\right) R$ are

$$
D_{1}=k\left((y / x)^{2}\right)+M \quad \text { and } \quad D_{2}=k\left((y / x)+(y / x)^{2}\right)+M,
$$

respectively, where $M$ is the maximal ideal of the ord-valuation domain $V=$ $R[y / x]_{\mathbf{m} R[y / x]}=k(y / x)+M$ over $R$. (The maximal ideals $M_{1}$ and $M_{2}$ of $D_{1}$ and $D_{2}$, respectively, are contained in $M$, and a module basis for $V$ over either $D_{1}$ or $D_{2}$ is $1, y / x$. Since $M_{i}(y / x) \subseteq D_{i}$ and $M_{i} V=M$, we have $M_{i}=M$.) Since $k$ is of characteristic zero, we have $k\left((y / x)^{2}\right) \cap k\left((y / x)+(y / x)^{2}\right)=k$. It follows that the residue field of $D_{1} \cap D_{2}$ at the center of $V$ on $D_{1} \cap D_{2}$ is not residually transcendental over the residue field $k$ of $R$, so $D_{1} \cap D_{2}$ is not a semispot over $R$ by (1.4) and hence is not the first coefficient domain of an ideal of $R$. 
(1.6) Suppose $I$ and $J$ are $\mathbf{m}$-primary ideals of $R$, where $(R, \mathbf{m})$ is a twodimensional RLR, or more generally, a quasi-unmixed analytically unramified local domain. We want to relate the first coefficient domain $D$ of $I J$ to the first coefficient domains $D_{I}$ and $D_{J}$ of $I$ and $J$. A first remark is that since the set of Rees valuation domains of $I J$ is the union of the sets of Rees valuation domains of $I$ and $J$, the integral closure of $D$ is the intersection of the integral closures of $D_{I}$ and $D_{J}$. With each DVR $V$ that is a localization of the integral closure of $D_{I}$ (of which there are only finitely many) we associate a one-dimensional semilocal domain $D_{V}=\left(D_{I}\right)_{P}[B]$, where $P$ is the center of $V$ on $D_{I}$ and $B$ is the unique local domain on the blowup of $J$ that is dominated by $V$. In an analogous way we construct $D_{W}$ for each DVR $W$ that is a localization of the integral closure of $D_{J}$. The first coefficient domain $D$ of $I J$ is the the intersection of the one-dimensional semilocal domains $D_{V}$ and $D_{W}$ as $V$ and $W$ vary over the sets of the Rees valuation domains of $I$ and $J$ respectively.

(1.7) The proofs of several results below rely on Theorem 3.12 of [HJL]; and on rereading the proof of that result, we feel one point deserves a fuller discussion. The relevant hypotheses in that result are as follows: $R$ is a normal, analytically unramified, quasi-unmixed, local domain with infinite residue field, $I$ is an ideal primary for the maximal ideal of $R, D$ is the first coefficient domain of $I, E$ is a domain birational and integral over $D$, and $a$ is an element of $I$ for which $I D=a D$. In the proof, we set $S=R[1 / a] \cap D$ and $T=R[1 / a] \cap E$, and we assert that $D, E$ are rings of fractions of $S, T$ respectively. This is true under the hypothesis of Theorem 3.12 of [HJL], but in Example 1.8 below we show that for $a \in \mathbf{m}$ with $a D \neq I D$ it can happen that $D$ is not a ring of fractions of $S=R[1 / a] \cap D$. So we felt these assertions should be given a more explicit justification: The hypothesis that $D$ is the first coefficient domain of $I$ means that there exists an element $b$ of $I$ such that $D$ is an intersection of a finite number of one-dimensional localizations of $R[I / b]$ and hence is itself a ring of fractions of $R[I / b]$. Moreover, $b D=$ $I D=a D$. Thus, $b / a$ is an element of $R[I / a]$ that is not in any of the prime ideals of $D$, so the ring of fractions of $R[I / a]$ with respect to the complement 
in $R[I / a]$ of the union of the primes in $D$ contains $R[I / b]$ and hence is all of $D$. Since $S=R[1 / a] \cap D \supseteq R[I / a]$, we see that $D$ is also a ring of fractions of $S$. Now we turn to $T=R[1 / a] \cap E$, which is almost integral over $S$ since there is a nonzero conductor from $E$ into $D$ (because $R$ is analytically unramified [Re2, Theorem 1.2]). Since

$$
S=\cap\left\{R[I / a]_{P}: P \text { is a height-one prime }\right\}
$$

and since $R[I / a]$ is universally catenary, $S$ is contained in the integral closure of $R[I / a]$. Moreover, the fact that $R$ is analytically unramified implies that the integral closure of $R[I / a]$ is a finitely generated $R[I / a]$-module. Therefore $S$ is Noetherian and hence $T$ is integral over $S$. Since $D$ is a ring of fractions of $S$, the maximal ideals of $D$ are centered on height-one primes of $S$. It follows that the maximal ideals of $E$ are centered on height-one primes of $T$. Since the essential valuation domains of $R[1 / a]$ are all localizations of $S$ and of $T$, it follows that $E$ is a ring of fractions of $T$.

Example 1.8. Let $R=k[x, y]_{(x, y) k[x, y]}$, where $k$ is a field and $x, y$ are indeterminates over $k$. Let $V=k(y / x)[x]_{(x)}$ be the ord-valuation domain of $R$. Then $V=k(y / x)+M$, where $M$ is the maximal ideal of $V$. Let $D=k\left(\left(y^{2}+x^{2}\right) / x y\right)+M$. Then $D$ is the first coefficient domain of the ideal $\left(x y, y^{2}+x^{2}\right) R$, a one-dimensional local domain that birationally dominates $R$, and $V$ is the integral closure of $D$. Let $T=R[1 / x] \cap V$ and $S=R[1 / x] \cap D$. Then $T=R[y / x]$, so $S=R[y / x] \cap D$. Using that $k[y / x] \cap k\left(\left(y^{2}+x^{2}\right) / x y\right)=k$ and considering the unique expression of each element of a subdomain of $V$ as the sum of an element of $k(y / x)$ and an element of $M$, we see that $S=k+(M \cap R[y / x])$. Hence $D$ is centered on a maximal ideal of $S$ and is not a localization of $S$. We also have in this example that $S$ is not Noetherian and $T$ is almost integral but not integral over $S$. The localization of $S$ at each of its height-one primes contains $R[1 / x]$.

\section{Residually transcendental elements.}

Let $(R, \mathbf{m})$ be a two-dimensional RLR with residue field $k=R / \mathbf{m}$. A first coefficient domain of an $\mathbf{m}$-primary ideal of $R$ is a one-dimensional semispot birationally dominating $R$. As a partial converse, we observe in 
Proposition 2.1 that a domain satisfying these hypotheses is at least a ring of fractions of a first coefficient domain of $R$.

Proposition 2.1. Let $(R, \mathbf{m})$ be a two-dimensional $R L R$ and $E$ be a onedimensional semispot birationally dominating $R$. Then there exists a first coefficient domain $D$ of $R$ such that $E$ is a ring of fractions of $D$.

Proof. Let $a_{1}, \ldots, a_{n}, b$ be elements of $R$ such that $E$ is a ring of fractions of $R\left[a_{1} / b, \ldots, a_{n} / b\right]$. We may assume that $a_{1}, \ldots, a_{n}, b$ have no common factor in $R$, so that the ideal $I=\left(a_{1}, \ldots, a_{n}, b\right) R$ is $\mathbf{m}$-primary. Let $D_{0}$ denote the first coefficient domain of $I$. Since $E$ is a semispot over $R$, the dimension formula [M, (14.D)] shows that for each maximal ideal $N$ of $E$ the image of at least one of the quotients $a_{i} / b$ in $E / N$ is transcendental over $R / \mathbf{m}$. Thus, the center of $N$ on $R\left[a_{1} / b, \ldots, a_{n} / b\right]$ is one-dimensional, so that $D_{0} \subseteq E_{N}$. Since this holds for each maximal ideal $N$ of $E, D_{0} \subseteq E$. But there may be prime divisors dominating $R$ that contain $D_{0}$ but not $E$. The intersection $D$ of all these prime divisors and $E$ is an integral extension of $D_{0}$ and hence a first coefficient domain (of an ideal integral over a power of $I$ ) by [HJL, Theorem 3.12]. We have $D \subseteq E$ are one-dimensional semilocal domains with $E$ birational over $D$ and $D$ integrally closed in $E$. Forming the ring of fractions of $D$ with respect to the elements of $D$ that are units of $E$ and applying $[\mathrm{N},(33.1)]$, we see that $E$ is a ring of fractions of $D$.

A variant of the process used in this proof is as follows: With $R, E$, etc. as in Proposition 2.1 and its proof, let $(c, d) R$ be a reduction of $I=$ $\left(a_{1}, \ldots, a_{n}, b\right) R$ (or of a power of $I$ if the residue field of $R$ is finite and $I$ fails to have a 2-generated reduction). For each maximal ideal $N$ of $E$, the image of $c / d$ in $E / N$ is transcendental over $R / \mathbf{m}$, so $N \cap R[c / d]=\mathbf{m} R[c / d]$. It follows that $E$ is a localization of the integral closure of $R[c / d]_{\mathbf{m} R[c / d]}$ in $E$. To realize $E$ itself as a first coefficient domain in this manner amounts to answering in the affirmative the following question: Does there exist a single element $a / b$ of $E$ such that $J=(a, b) R$ is a reduction of a complete ideal of the form $(*)$ in $(0.2)$ above, where the $r_{j}$ are positive integers and the $\mathbf{b}_{j}$ are the simple complete ideals corresponding to the DVR localizations of the integral closure of $E$ ? If so, then $E$ and $R[a / b]_{\mathbf{m} R[a / b]}$ have the same integral 
closure. Thus, $E$ is integral over $R[a / b]_{\mathbf{m} R[a / b]}$ and hence a first coefficient domain in its own right. The proof of Theorem 2.2 below is essentially the construction of such an element $a / b$ in a special case.

In the proof of Theorem 2.2 is a reference to $R(t)$, where $t$ is an indeterminate over $R$. In general, for a ring $A$, the symbol $A(t)$ denotes the ring of fractions of the polynomial ring $A[t]$ with respect to the multiplicative system of polynomials whose coefficients generate the unit ideal in $A$ (cf. [N, page 18]). In the present local case, this means only that not all of the coefficients of the polynomial are in $\mathbf{m}$. There is a natural epimorphism from $R(t)$ onto the simple transcendental field extension $k(t)$ of $k$, with kernel generated by $\mathbf{m}$; images under this epimorphism (as well as under other extensions of the epimorphism $R \rightarrow k$ ) are denoted by overbars (vincula).

Theorem 2.2. Let $D$ be a one-dimensional local domain birationally dominating a two-dimensional $R L R R$. Assume that $k=R / \mathbf{m}$ is algebraically closed, that the integral closure $D^{\prime}$ of $D$ is a prime divisor on $R$, and that either (1) $R$ has nonzero characteristic or (2) $D$ contains the maximal ideal of $D^{\prime}$. Then there is an $\mathbf{m}$-primary ideal of which $D$ is the first coefficient domain.

Proof. By (1.1), $D$ is a spot over $R$ and $D^{\prime}$ is a finitely generated $D$-module. In view of the last sentence of General Example 3.8 and Theorem 3.12 of [HJL], it is enough to find a 2-generated $\mathbf{m}$-primary ideal $(a, b) R$ of $R$ for which $a / b \in D$ and the integral closure of $R[a / b]_{\mathbf{m} R[a / b]}$ is $D^{\prime}$. Also, since $D^{\prime}$ is a prime divisor of the second kind of $R$, there is a simple complete $\mathbf{m}$-primary ideal $\mathbf{b}$ with which $D^{\prime}$ is associated, in the sense of the Zariski theory. It will suffice to find elements $a, b$ of $R$ so that $a / b \in D$ and the ideal $(a, b) R$ is a reduction of a power of $\mathbf{b}$.

Let $(c, d) R$ be a minimal reduction of $\mathbf{b}$ (or of a power of $\mathbf{b}$ ). Then the residue field of $D^{\prime}$ is of transcendence degree 1 over $k$, generated by the image $\overline{c / d}$ of $c / d$ (because $k$ is algebraically closed [HuS, Remark 3.5]), but algebraic over the residue field of $D$, and for any other prime divisor of the second kind of $R$, either $c / d$ is not in that prime divisor or its image in the residue field is not transcendental over the image of $k$ (i.e., $c / d$ is not 
"residually transcendental" for any other prime divisor of the second kind). Thus, for an element $z$ of $D$ of which the image $\bar{z}$ in the residue field of $D$ (or $D^{\prime}$ ) is transcendental over $k$, there is an element $\varphi(t)$ of $R(t)$ such that if $\bar{\varphi}(t) \in k(t)$ is the image of $\varphi(t)$ in $R(t) / \mathbf{m} R(t)$, then $\bar{z}=\bar{\varphi}(\overline{c / d})$. We may assume that the numerator and denominator of $\bar{\varphi}(t)$ are relatively prime polynomials over $k$. Now $z-\varphi(c / d)$ is in the maximal ideal of $D^{\prime}$, so under assumption (2) of the statement, we immediately have that $\varphi(c / d) \in D$. To reach a similar (though not identical) conclusion under assumption (1), we note that since $D^{\prime}$ is local and is a finitely generated $D$-module, the maximal ideal of $D$ contains a power of the maximal ideal of $D^{\prime}$; so we can raise $z-\varphi(c / d)$ to a sufficiently high power $q$, a power of the characteristic of $R$, to conclude that $\varphi(c / d)^{q} \in D$. Multiplying the numerator and denominator of $\varphi$ or $\varphi^{q}$ by the same power of $d$, we convert them into forms $a=a(c, d)$ and $b=b(c, d)$ in $c, d$ of the same degree $n$ such that their images in the degree- $n$ piece of the fiber ring $F((c, d))=R[(c, d) t] \otimes_{R} R / \mathbf{m}$, a polynomial ring in two variables over $k$, are relatively prime.

We show that $(a, b)$ is a reduction of $(c, d)^{n}$, which will complete the proof. It suffices to show that $(a, b)(c, d)^{n}=(c, d)^{2 n}$, and by Nakayama's Lemma it suffices to show that the $k$-vector spaces $\left[(a, b)(c, d)^{n}+\mathbf{m}(c, d)^{2 n}\right] / \mathbf{m}(c, d)^{2 n}$ and $(c, d)^{2 n} / \mathbf{m}(c, d)^{2 n}$ have the same dimension. The latter is the degree- $2 n$ piece of the fiber ring $F((c, d))$; its dimension is $2 n+1$. The images of the products $a c^{i} d^{n-i}, i=0, \ldots, n$, span a subspace of the former of dimension $n+1$, and similarly with $b$ in place of $a$; and since the images of $a, b$ are relatively prime, the intersection of these two subspaces is spanned by the image of $a b$, so it is one-dimensional. Thus, $\left[(a, b)(c, d)^{n}+\mathbf{m}(c, d)^{2 n}\right] / \mathbf{m}(c, d)^{2 n}$ has dimension $2(n+1)-1=2 n+1$ as required.

\section{Principal extensions and contracted powers.}

(3.1) Suppose $D$ is a one-dimensional semispot birationally dominating a quasi-unmixed, analytically unramified, normal local domain $(R, \mathbf{m})$. In this section we seek conditions for $D$ to be the first coefficient domain of an ideal $I$ of $R$. If $D$ is the first coefficient domain of $I$, then $I D$ is principal, and replacing $I$ by the associated $e_{1}$-ideal of a high power of $I$, we obtain 
an $\mathbf{m}$-primary ideal $J$ such that $J D$ is principal and $J^{n} D \cap R=J^{n}$ for each positive integer $n$ [HJLS, Theorem 3.17]. Thus a necessary condition for $D$ to be a first coefficient domain is the existence of an $\mathbf{m}$-primary ideal $J$ of $R$ with the two properties: (1) $J D$ is principal, and (2) $J^{n} D \cap R=J^{n}$ for each positive integer $n$. If $D$ is local, we prove in Theorem 3.3 that this necessary condition is also sufficient, and that $D$ is in fact the first coefficient domain of each ideal $J$ with these two properties.

The case in which $V$ is a prime divisor birationally dominating a twodimensional $\operatorname{RLR}(R, \mathbf{m})$ is illustrative. Suppose $a$ is a nonzero element of $\mathbf{m}$ and consider the descending chain $J_{n}=a^{n} V \cap R, n=1,2, \ldots$, of ideals of $R$. As noted in the introduction, each $J_{n}$ is a complete ideal of $R$, and from the Zariski theory it follows that $J_{n}$ is a product of powers of the simple complete ideals associated with the finitely many prime divisors that "come out" on the sequence of quadratic transformations of $R$ along $V$. Let $\mathbf{b}$ be the simple complete ideal of $R$ associated to $V$, and suppose the $V$-values of $a$ and $\mathbf{b}$ are $p$ and $q$ respectively. Then $J_{q}=\mathbf{b}^{p}$. Since all powers of b are contracted from $V$, for each positive integer $r$ we have $J_{q}^{r}=J_{q r}$, or equivalently the powers of $J_{q}$ are contracted from $V$. Moreover, $J_{q}$ has $V$ as its first coefficient domain.

(3.2) It was noted in [HJL, (3.7)] that the first coefficient domain of an ideal $I$ of $R$ can be described using the minimal primes of $I R[I t]$ of the Rees algebra $R[I t]$ or the minimal primes of $t^{-1} R\left[t^{-1}, I t\right]$ of the extended Rees algebra $R\left[t^{-1}, I t\right]$ of $I$ (where $t$ is an indeterminate over $R$ ). These primes are in one-to-one correspondence with the maximal ideals of the first coefficient domain $D$ of $I$ : If $P$ is one of these minimal primes, then $P$ does not contain the degree-1 piece of the Rees algebra (or extended Rees algebra), say $b t \notin P$ where $b \in I$. Then the localization of the (extended) Rees algebra at $P$ is also a localization of $R[I / b]\left[b t,(b t)^{-1}\right]$ and has the form $D_{N}(b t)$ (cf. the paragraph before Theorem 2.2) for the maximal ideal $N$ of $D$ corresponding to $P$. [Note: The $V(t)$ in the equations on the last line of [HJL, (3.7)] should be $V(b t)$, for $b$ as above.]

Theorem 3.3. Let $(R, \mathbf{m})$ be a local domain that is the intersection of its 
localizations at height-one primes, and let $D$ be a one-dimensional semilocal domain that birationally dominates $R$. Suppose $J$ is an $\mathbf{m}$-primary ideal of $R$ such that $J D$ is principal and $J^{n} D \cap R=J^{n}$ for each positive integer $n$. Then the first coefficient domain of $J$ is a localization of $D$. In particular, if $D$ is local, then $D$ is the first coefficient domain of $J$.

Proof. Replacing $J$, if necessary, by a power of $J$, we may assume that $J D=a D$ where $a \in J$. Let $A=R\left[t^{-1}, J t\right]$ be the extended Rees algebra of the ideal $J$ of $R$; let $D(a t)$ denote the localization of the polynomial ring $D[a t]$ at the complement of the union of the extension to $D[a t]$ of the maximal ideals of $D$; and let $K$ be the fraction field of $R$. Since $D\left[a t,(a t)^{-1}\right]$ is Cohen-Macaulay, it is the intersection of its localizations at height-one primes. It follows that $D\left[a t,(a t)^{-1}\right]=K\left[a t,(a t)^{-1}\right] \cap D(a t)$, and hence that

$$
\begin{aligned}
R\left[t, t^{-1}\right] \cap D\left[a t,(a t)^{-1}\right] & =R\left[t, t^{-1}\right] \cap K\left[a t,(a t)^{-1}\right] \cap D(a t) \\
& =R\left[t, t^{-1}\right] \cap D(a t)=A .
\end{aligned}
$$

Let $P$ be a minimal prime of $t^{-1} A$ and let $S=A-P$. Then $A_{P}=$ $S^{-1}\left(R\left[t, t^{-1}\right] \cap D(a t)\right)=S^{-1}\left(R\left[t, t^{-1}\right]\right) \cap S^{-1} D(a t)$. Since $R\left[t, t^{-1}\right]$ is the locally finite intersection of its localizations at height-one primes, to show $S^{-1}\left(R\left[t, t^{-1}\right]\right)=K(t)$, it suffices to show $S$ meets each height-one prime $Q$ of $R\left[t, t^{-1}\right]$ : If $Q \cap S=\emptyset$, then $Q \cap A \subseteq P$. Since $Q \cap A \neq 0$, we must have $Q \cap A=P$. But $P \cap R=\mathbf{m}$ and $Q \cap R<\mathbf{m}$, a contradiction. Thus $S$ meets each height-one prime of $R\left[t, t^{-1}\right]$, so $A_{P}=S^{-1} D(a t)$.

Let $E$ be the first coefficient domain of $J$. The maximal ideals $N$ of $E$ are in one-to-one correspondence with the minimal primes $P$ of $t^{-1} A$, where $A_{P}=E_{N}(a t)$. Since each $A_{P}$ is a localization of $D(a t)$, the intersection $E(a t)$ of the $A_{P}$ 's is a ring of fractions of $D(a t)$. Intersecting with $K$ shows that $E$ is a ring of fractions of $D$.

The following corollary implies the uniqueness property of the intersection of the Rees valuation domains of an ideal mentioned in (0.2).

Corollary 3.4. Let $(R, \mathbf{m})$ be a quasi-unmixed, analytically unramified, normal local domain, and let $I$ be an $\mathbf{m}$-primary ideal of $R$. The first coefficient 
domain $E$ of $I$ is the unique largest one-dimensional semilocal domain $D$ birationally dominating $R$ and having the properties that ID is principal and $I^{n} D \cap R$ is contained in the $e_{1}$-ideal of $I^{n}$ for each positive integer $n$.

Proof. By [HJLS, Theorem 3.17] for all sufficiently large positive integers $r$, the ideal $J=I^{r} E \cap R$ has the property that $E$ is the first coefficient domain of $J$ and for each positive integer $n$ we have $J^{n} E \cap R=J^{n}=I^{r n} E \cap R$ is the $e_{1}$-ideal associated to $I^{r n}$. Therefore $J^{n} D$ is principal and $J^{n} D \cap R=J^{n}$ for each $n$. By Theorem 3.3, $E$ is a localization of $D$.

Corollary 3.5. Let $D$ be a one-dimensional spot birationally dominating a two-dimensional $R L R(R, \mathbf{m})$. If $J$ is an $\mathbf{m}$-primary ideal in $R$ such that $J D$ is principal and all the powers of $J$ are contracted from $D$, then $D$ is the first coefficient domain of $J$, and the integral closure of $J$ is a product of powers of the simple complete ideals associated to the localizations of the integral closure of $D$.

Proposition 3.6. Let $(R, \mathbf{m})$ be a quasi-unmixed analytically unramified local domain of dimension $d \geq 2$, and let $J$ be an $\mathbf{m}$-primary ideal of $R$. Let $D$ be a one-dimensional semilocal domain birationally dominating $R$, and let $V$ be a finitely generated birational integral extension of $D$. If all the powers of $J$ are contracted from $D$, then for each positive integer $n, J^{n} V \cap R$ is integral over $J^{n}$. In particular, if $I=J D \cap R$ is a normal ideal (i.e., the powers of $I$ are integrally closed), then all the powers of $I$ are contracted from $V$.

Remark. The hypothesis in Proposition 3.6 (and in Proposition 3.7 below) that $V$ is a finitely generated $D$-module is necessary (cf., e.g., [HRS, (1.27)]). But if $D$ (or $V$, by Corollary 1.3) is a (birational) semispot over $R$, the hypothesis on $R$ assures that $V$ is a finitely generated $D$-module [Re2, Theorem 1.2].

Proof. For the first assertion, it suffices to show that $I$ is integral over $J$. Since $J$ is contained in each nonzero prime ideal of $D$, there exists a positive integer $c$ such that $J^{c}$ is contained in the conductor of $V$ into $D$. Thus, for 
all positive integers $n$, we have

$$
I^{n+c} \subseteq I^{n+c} V \cap R=J^{n+c} V \cap R \subseteq J^{n} D \cap R=J^{n} \subseteq I^{n} .
$$

It follows that the length of $R / J^{n}$ is between those of $R / I^{n}$ and $R / I^{n+c}$. Now, for $n$ sufficiently large, the length of $I^{n} / I^{n+c}$ is a polynomial in $n$ of degree $d-1$, while the lengths of $R / I^{n}$ and $R / J^{n}$ are polynomials in $n$ of degree $d$. Therefore the Hilbert polynomials of $I$ and $J$ have the same highest degree coefficient, i.e., $I$ and $J$ have the same multiplicity. By $[\operatorname{Re} 1$, Theorem 3.2], $I$ is integral over $J$.

For the second assertion, note that $J^{n} \subseteq I^{n} \subseteq I^{n} V \cap R=J^{n} V \cap R$; the last ideal is integral over $J^{n}$, so if $I^{n}$ is integrally closed, it is equal to $I^{n} V \cap R$.

Proposition 3.7. Let $(R, \mathbf{m})$ be a normal, quasi-unmixed, analytically unramified local domain of dimension $d \geq 2$, and $J$ be an $\mathbf{m}$-primary ideal of $R$. Let $D$ be a one-dimensional semilocal domain birationally dominating $R$ such that the integral closure $V$ of $D$ is a finitely generated D-module. Suppose that $J^{n} D \cap R=J^{n}$ for each positive integer $n$, and let $I_{n}=J^{n} V \cap R$ for each $n$.

(1) For sufficiently large $r$, all the powers of $I_{r}$ are contracted from $V$.

(2) Therefore $V$ is contained in each of the Rees valuation domains of $J$, and so $D$ is contained in the integral closure of the first coefficient domain of $J$.

Proof. (1) By [Re2, Theorem 1.4], for sufficiently large $r, I_{r}$ is a normal ideal, so by Proposition 3.6 all the powers of $I_{r}$ are contracted from $V$. (2) Since the intersection of the Rees valuation domains is the unique largest one-dimensional semilocal subdomain $E$ of the fraction field of $R$ with the property that the integral closure of $J^{n}$ is $J^{n} E \cap R$ for each $n, V$ is contained in each of the Rees valuation domains of $J$.

(3.8) Let $(R, \mathbf{m})$ be a two-dimensional RLR, let $D$ be a one-dimensional semispot birationally dominating $R$, and let $V$ be the integral closure of $D$. Let $\mathbf{b}_{1}, \ldots, \mathbf{b}_{n}$ be the simple complete ideals of $R$ associated to the DVR's 
which are localizations of $V$. Then the associated $e_{1}$-ideal of an $\mathbf{m}$-primary ideal $I$ of $R$ has the form $(*)$ as in $(0.2)$ above, where the $r_{j}$ are positive integers, if and only if $V$ is the first coefficient domain of $I$. By [HJL, Theorem 3.12], $D$ is a first coefficient domain if and only if there exists an ideal $J$ of $R$ such that $J D$ is principal and such that the integral closure of $J$ is of the form $(*)$. Thus, for example, if $V$ is the ord-valuation domain of $R$, then $D$ is a first coefficient domain if and only if there exists an ideal $J$ such that $J D$ is principal and such that the integral closure of $J$ is a power of $\mathbf{m}$.

(3.9) With $R, D$ as in Corollary 3.5, there always exist $\mathbf{m}$-primary ideals $J$ with the property that all their powers are contracted from $D$ (for, if $J$ is the product of the simple complete ideals associated to the DVR localizations of the integral closure of $D$, then all the powers of $J$ are contracted from the integral closure of $D$ and hence also from $D$ ). Thus, in this case the issue is whether there exists such a $J$ with $J D$ principal. However, if one passes to a more general situation where $R$ is a two-dimensional excellent normal local domain, then there may exist birationally dominating DVR spots $V$ over $R$ for which there does not exist an ideal $J$ of $R$ such that all the powers of $J$ are contracted from $V$. By definition, an excellent two-dimensional normal local domain $(R, \mathbf{m})$ with the property that each prime divisor of the second kind on $R$ is the first coefficient domain of an $\mathbf{m}$-primary ideal is said to satisfy Muhly's condition $(N)$ (cf. [HL, page 291]). If $R$ is a two-dimensional complete normal local domain, Cutkosky proves in [C, Theorem 4] that $R$ satisfies condition $(N)$ if and only if $R$ has torsion divisor class group. Thus, for example, $R=\mathbb{C}[[x, y, z]]$, where $x^{3}+y^{3}+z^{3}=0$, has prime divisors of the second kind which are not first coefficient domains of an ideal of $R$.

(3.10) Let $(R, \mathbf{m})$ be a two-dimensional RLR and let $D$ be the first coefficient domain of an ideal $I$ of $R$. If $D$ is a prime divisor of $R$ and $a \in \mathbf{m}$ is a nonzero element, then there exists a positive integer $n$ such that $D$ is the first coefficient domain of $a^{n} D \cap R$ (cf. (3.1)). The case of a general first coefficient domain, however, is different: In Example 1.8, there is no positive integer $m$ for which $D$ is the first coefficient domain of $x^{m} D \cap R$. This 
phenomenon is the reef on which founders the following naive approach to realizing a one-dimensional semispot $E$ birationally dominating $R$ as a first coefficient domain. Let $\mathbf{b}_{1}, \ldots, \mathbf{b}_{s}$ be the distinct simple complete ideals of $R$ associated with the prime divisors obtained as localizations of the integral closure $E^{\prime}$ of $E$, and let $a \in R$ be such that $a E^{\prime} \cap R=\mathbf{b}_{1} \ldots \mathbf{b}_{s}$. Let $A=R\left[t^{-1}, t\right] \cap E(a t)$. Then $A=R\left[t^{-1}, I_{1} t, I_{2} t^{2}, \ldots\right]$, where $I_{n}=a^{n} E \cap R$. The integral closure of $A$ is $A^{\prime}=R\left[t^{-1},\left(I_{1}\right)^{\prime} t,\left(I_{2}\right)^{\prime} t^{2}, \ldots\right]$, while the domain $A^{\prime \prime}=R\left[t^{-1}, t\right] \cap E^{\prime}(a t)$ is almost integral over $A$ since there is a nonzero conductor from $E^{\prime}$ to $E$. The following conditions are equivalent: (1) $A$ is Noetherian. (2) $A$ is affine over $R$. (3) $A^{\prime}=A^{\prime \prime}$. When these conditions hold, $\left(I_{1}\right)^{\prime}=\mathbf{b}_{1} \ldots \mathbf{b}_{s}$ and $E$ is the first coefficient domain of an ideal integral over a power of $I_{1}$. In Example 1.8, however, for $E=D$ and $a=x$, we have $A^{\prime}<A^{\prime \prime}$. When we have $A^{\prime}<A^{\prime \prime}$, there is no positive integer $m$ for which the powers of $I_{m}$ are contracted from $E$, nor for which $E$ is the first coefficient domain of $I_{m}$.

\section{ACKNOWLEDGEMENT}

This work is the outgrowth of a project with Bernard Johnston. We would like to acknowledge his penetrating questions which initiated the present paper. We also thank the referee for a careful reading of the paper.

\section{REFERENCES}

[A1] S. Abhyankar, On the valuations centered in a local domain, Amer. J. Math. 78 (1956), 321-348.

[A2] S. Abhyankar, Quasi-rational singularities, Amer. J. Math. 100 (1978), 267-300.

[C] S. Cutkosky, On unique and almost unique factorization of complete ideals II, Invent. Math. 98 (1989), 59-74.

[HJL] W. Heinzer, B. Johnston and D. Lantz, First coefficient domains and ideals of reduction number one, Comm. Algebra 21 (1993), 3797-3827.

[HJLS] W. Heinzer, B. Johnston, D. Lantz and K. Shah, Coefficient ideals in and blowups of a commutative Noetherian domain, J. Algebra 162 (1993), 355-391.

[HL] W. Heinzer and D. Lantz, Exceptional prime divisors of two-dimensional local domains, Commutative Algebra: Proceedings of a Microprogram Held June 15July 2, 1987, Springer-Verlag, New York, 1989.

[HRS] W. Heinzer, C. Rotthaus and J. Sally, Formal fibers and birational extensions, Nagoya Math. J. 131 (1993), 1-38.

[Hu] C. Huneke, Complete ideals in two-dimensional regular local rings, Commutative 
Algebra: Proceedings of a Microprogram Held June 15-July 2, 1987, SpringerVerlag, New York, 1989.

[HuS] C. Huneke and J. Sally, Birational extensions in dimension two and integrally closed ideals, J. Algebra 115 (1988), 481-500.

[Ku] E. Kunz, Introduction to Commutative Algebra and Algebraic Geometry, Birkhäuser, Boston, 1985.

[M] H. Matsumura, Commutative Algebra, Second Edition, Benjamin/Cummings, Reading, Massachusetts, 1980.

[N] M. Nagata, Local Rings, Interscience, New York, 1962.

[No] S. Noh, Sequence of valuation ideals of prime divisors of the second kind in 2dimensional regular local rings, J. Algebra 158 (1993), 31-49.

[Re1] D. Rees, a-transforms of local rings and a theorem on multiplicities of ideals, Proc. Cambridge Phil. Soc. 57 (1961), 8-17.

[Re2] D. Rees, A note on analytically unramified rings, J. London Math. Soc. 36 (1961), 24-28.

[Sh] K. Shah, Coefficient ideals, Trans. Amer. Math. Soc. 327 (1991), 373-384.

[Z] O. Zariski, Polynomial ideals defined by infinitely near base points, Amer. J. Math. 60 (1938), 151-204.

[ZS] O. Zariski and P. Samuel, Commutative Algebra, Vol. II, Springer-Verlag, New York, 1975. 